INPLASY

PROTOCOL

To cite: Xiao et al. Efficacy of

Xiang-Sha-Liu-Jun-Zi on chemotherapy-induced nausea and vomiting: A protocol for systematic review and metaanalysis. Inplasy protocol

202140079. doi:

10.37766/inplasy2021.4.0079

Received: 14 April 2021

Published: 14 April 2021

Corresponding author: Hang Xiao

1206811542@qq.com

Author Affiliation: Jiangxi University of Traditional Chinese Medicine

Support: Grant No. 81860826.

Review Stage at time of this submission: Preliminary searches.

Conflicts of interest: None declared.

\section{Efficacy of Xiang-Sha-Liu-Jun-Zi on chemotherapy-induced nausea and vomiting: A protocol for systematic review and meta-analysis}

Xiao, H1; Liu, LJ2; Ke, SW3; Zhang, YQ4; Zhang, WQ5; Xiong, SB6; Zhang, W7; Ouyang, JQ8.

Review question / Objective: Efficacy of Xiang-Sha-Liu-Jun-Zi on chemotherapy-induced nausea and vomiting.

Condition being studied: Cancer is the main cause of death worldwide, and chemotherapy is the basic method of treating cancer. However, chemotherapy-induced nausea and vomiting (CINV) is the most common side effect of chemotherapy, and conventional antiemetics for the treatment of CINV also have side effects. At present, a large number of randomized controlled trials (RCTs) have shown that Xiang-Sha-Liu-Jun-Zi (XSLJZ) can effectively treat CINV, but there is no systematic review. Therefore, this systematic review aims to discuss the effectiveness of XSLJZ in the treatment of CINV.

INPLASY registration number: This protocol was registered with the International Platform of Registered Systematic Review and Meta-Analysis Protocols (INPLASY) on 14 April 2021 and was last updated on 14 April 2021 (registration number INPLASY202140079).

\section{INTRODUCTION}

Review question / Objective: Efficacy of Xiang-Sha-Liu-Jun-Zi on chemotherapyinduced nausea and vomiting.

Condition being studied: Cancer is the main cause of death worldwide, and chemotherapy is the basic method of treating cancer. However, chemotherapyinduced nausea and vomiting (CINV) is the most common side effect of chemotherapy, and conventional antiemetics for the treatment of CINV also have side effects. At present, a large number of randomized controlled trials (RCTs) have shown that 
Xiang-Sha-Liu-Jun-Zi (XSLJZ) can effectively treat CINV, but there is no systematic review. Therefore, this systematic review aims to discuss the effectiveness of XSLJZ in the treatment of CINV.

\section{METHODS}

Participant or population: For patients who are clearly diagnosed with cancer through pathological or cytological examination, the type of cancer, pathological type and stage are not limited. At the same time, liver and kidney indicators are basically normal, there is no contraindication to chemotherapy, and symptoms of CINV. Age and gender are not restricted.

Intervention: The treatment group was treated with XSLJZ (decoction, granule, tablet and powder) combined with chemotherapy.

Comparator: The control group received conventional chemotherapy.

Study designs to be included: (1) Type of studies: RCTs, whether using blinding. (2) Type of participants: For patients who are clearly diagnosed with cancer through pathological or cytological examination. (3) Types of interventions: The treatment group was treated with XSLJZ (decoction, granule, tablet and powder) combined with chemotherapy. (4) Type of comparators: The control group received conventional chemotherapy.

Eligibility criteria: (1) Non-RCT documents. (2) Duplicate documents. (3) The research data is incomplete or there is no full text. (4) Effective outcome data cannot be extracted from the literature. (5) Documents that are not in line with the subject direction of this research.

Information sources: First, we import all the searched literature transcripts into Endnote20.0 software, and use it to eliminate duplicate research. Secondly, after deleting the duplicate research literature, two authors independently reviewed the subject, abstract, and full text of the literature, and screened them according to the inclusion and exclusion criteria. Finally, the documents with discrepancies in the review will be reviewed and decided by the third author.

Main outcome(s): The effective rate of treatment is the main outcome indicator of this study.

Additional outcome(s): The secondary indicators of this study include the incidence of adverse reactions and the improvement rate of quality of life.

Quality assessment / Risk of bias analysis: Heterogeneity assessment is to determine whether there are differences between independent studies. Chi-square test and 12 test are used to test the heterogeneity of the included literature. If $P>0.1$ and $12<50 \%$, there is no heterogeneity in the independent study. The data were merged and a fixed effects model was used for meta-analysis. On the contrary, when P50\%, independent studies are heterogeneous, and the data are merged and a random effects model is used for meta-analysis.

Strategy of data synthesis: We will use Revman 5.3 software for meta-analysis. If it is continuous data, the mean difference (MD) will be used as the combined effect statistic and its $95 \%$ confidence interval (CI) will be calculated. However, for binary data, odds ratio (OR) will be used as the combined effect statistic and its $95 \% \mathrm{Cl}$ will be calculated.

Subgroup analysis: If the heterogeneity is found to be large in the heterogeneity test, a subgroup analysis is performed according to the cancer type.

Sensitivity analysis: Revman 5.3 software was used for sensitivity analysis to evaluate the reliability of meta-analysis. If the heterogeneity is high, we need to exclude low-quality or small sample research literature, and then perform the meta-analysis again to compare the results with the non-exclusive meta-analysis. If the 
result is basically stable, it is considered credible.

Country(ies) involved: China.

Keywords: chemotherapy, nausea and vomiting, Xiang-Sha-Liu-Jun-Zi, protocol, systematic review.

Contributions of each author:

Author 1 - Hang Xiao.

Email: 1206811542@qq.com

Author 2 - Liangji Liu.

Email: Ilj6505@163.com

Author 3 - Shiwen Ke.

Email: ksw14556@sina.com

Author 4 - Yuqin Zhang.

Email: 1843089974@qq.com

Author 5 - Wenqiang Zhang.

Email: 1014316416@qq.com

Author 6 - Shaobin Xiong.

Email: 1490748223@qq.com

Author 7 - Wei Zhang.

Email: 757886690@qq.com

Author 8 - Jiaqing Ouyang.

Email: 1029948456@qq.com 